\title{
Short- and long-term outcomes of definitive chemoradiotherapy in patients with esophageal carcinoma aged $\geq 75$ years
}

\author{
XIAOXU LU, HUI WU, JIANHUA WANG and JING XU \\ Department of Radiotherapy, Affiliated Tumor Hospital of Zhengzhou University, \\ Henan Tumor Hospital, Jinshui, Henan 45003, P.R. China
}

Received August 14, 2013; Accepted December 9, 2013

DOI: $10.3892 / \mathrm{mco} .2013 .235$

\begin{abstract}
The most relevant treatment modalities in elderly patients with esophageal carcinoma (EC) remain a subject of debate. Combined definitive chemoradiotherapy (CRT) is currently widely accepted as a non-surgical treatment for esophageal cancer. However, elderly patients were excluded from the majority of studies on CRT, or the proportion of elderly patients was relatively low in those studies and the number of available studies on the treatment of EC patients aged $\geq 75$ years is limited. The aim of the present retrospective study was to analyze the safety and efficacy of CRT in patients aged $\geq 75$ years in order to assess the short- and long-term outcomes of CRT for elderly patients with EC. In this study, based on further refinement of patient age groups and analysis of the Charlson comorbidity score, we performed a statistical analysis of factors such as short-term response, long-term survival and toxicity reactions. The results of the analysis indicated that the treatment of patients with EC aged $\geq 75$ years with radiotherapy (RT) and chemotherapy was effective. However, we recommend that customized treatment is based on the stratification of patients into different age groups and the Charlson score, as for patients aged $\geq 80$ years a lower-dose therapy may be more beneficial and for patients aged $\geq 85$ years definitive CRT should be administered with greater caution.
\end{abstract}

Correspondence to: Dr Hui Wu, Department of Radiotherapy, Affiliated Tumor Hospital of Zhengzhou University, 127 Dongming Road, Zhengzhou, Henan 450008, P.R. China

E-mail:wuhui7008@126.com

Abbreviations: CRT, chemoradiotherapy; RT, radiotherapy; EC, esophageal carcinoma; 5FU-CDDP, 5-fluorouracil-cisplatin; $\mathrm{CR}$, complete response; OS, overall survival; uRT, unfinished radiotherapy; cRT, completed radiotherapy; dCRT, discontinued chemoradiotherapy; rCRT, reduced chemoradiotherapy; cCRT, completed chemoradiotherapy.

Key words: esophageal carcinoma, definitive chemoradiotherapy, elderly

\section{Introduction}

Clinical trials since the 1990s have demonstrated that combined definitive chemoradiotherapy (CRT) is superior to radiotherapy (RT) alone for the treatment of esophageal carcinoma (EC) $(1,2)$. However, the most relevant treatment modalities in elderly patients with EC remain a subject of debate. It was reported that elderly patients are less likely to undergo surgery and chemotherapy, which is partially due to their comorbidities $(3,4)$. Currently, CRT based on the 5-fluorouracil-cisplatin (5FU-CDDP) regimen is used for the treatment with curative intent of locally advanced or inoperable non-metastatic EC (5).

The number of available studies on the safety and efficacy of CRT in elderly patients with locally advanced EC is currently limited, with a consequent lack of sufficient data regarding tolerance and short- and long-term outcomes of CRT in such patients. In two previous randomized trials that investigated the efficacy of CRT, the proportion of patients aged $\geq 75$ years was 34.9 and $45 \%(6,7)$, which was not considered sufficient to provide adequate reference data for the treatment of patients in this age group.

Thus, we conducted a retrospective analysis in a single institution to determine the safety and efficacy of CRT in EC patients aged $\geq 75$ years and assess the short- and long-term outcomes of CRT in such patients.

\section{Materials and methods}

Patient inclusion. The medical records of a total of 312 EC patients aged $\geq 75$ years who had undergone non-surgical treatment at the Affiliated Tumor Hospital of Zhengzhou University (Jinshui, China) between January, 2002 and March, 2008 were retrospectively evaluated. The study protocol was approved by a suitably constituted Ethics Committee of the Affiliated Tumor Hospital of Zhengzhou University and conformed to the provisions of the Declaration of Helsinki, 1995 (as revised in Tokyo, 2004). All patients had histologically confirmed squamous EC, without distant metastases at the time of diagnosis. The general condition of the patients was evaluated using the Eastern Cooperative Oncology Group (ECOG) performance status (PS) criteria and patients with an ECOG PS of $\geq 4$ were excluded from the study. We computed a comorbidity score for each patient using the variables included in the Charlson comorbidity index 
(myocardial infarction, congestive heart failure, peripheral vascular disease, cerebrovascular disease, dementia, chronic pulmonary disease, connective tissue disease, peptic ulcer disease, mild to severe liver disease, diabetes with/without end-organ damage, hemiplegia, moderate or severe renal disease and AIDS). Each condition was weighted and the patients were assigned a score based on the Charlson index (8).

Tumor staging. According to the TNM classification of the International Union Against Cancer in 2003, clinical staging was based on esophagography, esophagoscopy, color Doppler and computed tomography of the neck, chest and abdomen. Endoscopic ultrasonography, magnetic resonance imaging, bronchofiberscopy, ultrasonography or bone scintigraphy were additionally performed for individual staging.

Treatments. All patients underwent conventional two-dimensional RT or three-dimensional conformal RT. The treatment details were described previously (6). RT was performed with 6 or $8 \mathrm{MV}$ linear accelerators using a standard fractionation regimen (1.8-2.0 Gy/fraction; 5 days/week) at a prescribed dose of 50-64 Gy.

Concurrent chemotherapy consisted of CDDP at a dose of $60 \mathrm{mg} / \mathrm{m}^{2}$ on days $1-3$ administered by bolus injection and 5 -FU at a dose of $400 \mathrm{mg} / \mathrm{m}^{2}$ on days $1-5$ administered by continuous infusion. These chemotherapy regimens consisted of three courses administered every 3 weeks. Weekly or more frequent complete blood counts were recorded and the doses of 5-FU and CDDP were reduced by $20 \%$ if the white blood cell count was $2,500-3,500$ on day 21 .

Short-term response evaluation criteria. Evaluation of the short-term response of elderly EC patients to CRT was performed using the short-term Response Evaluation Criteria in Solid Tumors established by Therase et al (9) and based on X-ray film obtained following an esophageal barium meal. Local therapeutic failure refers to no changes in the esophageal lesions or progressive disease following RT and chemotherapy. Recurrence refers to the progression of esophageal lesions after achieving a complete response (CR) or partial response (PR) following RT and chemotherapy, reappearance of local lesions after 6 months and appearance of new lesions outside of the irradiation field.

Toxicity scoring criteria. Radiation toxicity was scored using the Radiation Therapy Oncology Group criteria (10), which included acute reactions occurring within the first 90 days of treatment, or late reactions occurring after 90 days of treatment. Chemotherapy-related toxicity was scored using the National Cancer Institute Common Toxicity Criteria, version 2.0 (11), and tabulated continually over the course of treatment. The most severe reaction was recorded as the toxicity score for the entire treatment.

Follow-up and statistical analysis. Patients were monitored weekly during the course of RT and every 3-6 months following the completion of treatment. Patient follow-up was conducted using outpatient records, ward records and telephone interviews. All endpoints were observed from the first day of treatment until death or the last follow-up contact.
Table I. Patients characteristics.

\begin{tabular}{lrr}
\hline Characteristics & $\begin{array}{c}\text { Patient no. } \\
(\mathrm{n}=312)\end{array}$ & $\%$ \\
\hline $\begin{array}{l}\text { Age range, years } \\
\text { (mean } \pm \text { SD })\end{array}$ & & \\
$75-79(76.9 \pm 1.4)$ & 193 & 61.86 \\
$80-84(81.3 \pm 1.4)$ & 88 & 28.20 \\
$85-89(86.5 \pm 1.1)$ & 24 & 7.70 \\
$\geq 90 \quad(94.9 \pm 6.2)$ & 7 & 2.24 \\
Performance status & & \\
0 & 60 & 19.2 \\
1 & 179 & 57.4 \\
$\geq 2$ & 73 & 23.4 \\
Charlson score & & \\
0 & 42 & 13.5 \\
1 & 146 & 46.8 \\
$\geq 2$ & 124 & 39.7 \\
T stage & & \\
T1 & 44 & 14.1 \\
T2 & 149 & 47.8 \\
T3 & 64 & 20.5 \\
T4 & 55 & 17.6 \\
N stage & & \\
N0 & 129 & 51.3 \\
N1 & 177 & 26.0 \\
TNM stage & & \\
I & & \\
II & & \\
III & 135.7 \\
\hline
\end{tabular}

SD, standard deviation; TNM, tumor-node-metastasis.

Statistical analysis was performed using the $\chi^{2}$ test for two-proportion comparisons. The survival rates were calculated from the date of treatment initiation until death, or the last follow-up contact for surviving patients, using the Kaplan-Meier method. The differences between survival rates were assessed using the log-rank test. $\mathrm{P}<0.05$ was considered to indicate a statistically significant difference. All analyses were performed using SPSS for Windows, version 18.0 (SPSS, Inc., Chicago, IL, USA).

\section{Results}

Patient and tumor characteristics. A total of 312 patients with locally advanced EC, aged $\geq 75$ years, who were treated between January, 2002 and March, 2008, were included in this study. The patient age ranged from 75 to 107 years (median, 79 years) and 31 of the patients $(9.9 \%)$ were aged $\geq 85$ years. The patient sample included 118 women and 194 men. The prevalence of comorbidity, defined as a Charlson score of $\geq 1$, was $86.5 \%$. The mean total RT dose delivered was $50.7 \mathrm{~Gy}$. The patient and tumor baseline characteristics are summarized in Table I. 
Table II. Patient stratification by clinical stage, treatment modality and treatment completion.

\begin{tabular}{|c|c|c|c|c|c|}
\hline \multirow[b]{2}{*}{ Stage } & \multicolumn{3}{|c|}{ CRT } & \multicolumn{2}{|c|}{ RT } \\
\hline & cCRT & rCRT & dCRT & cRT & uRT \\
\hline \multicolumn{6}{|l|}{$\mathrm{T}$} \\
\hline $\mathrm{T} 1$ & 13 & 6 & 5 & 16 & 4 \\
\hline $\mathrm{T} 2$ & 47 & 22 & 22 & 44 & 14 \\
\hline T3 & 21 & 9 & 8 & 19 & 7 \\
\hline $\mathrm{T} 4$ & 18 & 10 & 7 & 15 & 5 \\
\hline \multicolumn{6}{|l|}{$\mathrm{N}$} \\
\hline N0 & 55 & 26 & 23 & 52 & 21 \\
\hline N1 & 44 & 21 & 19 & 42 & 9 \\
\hline \multicolumn{6}{|l|}{ TNM } \\
\hline I & 31 & 15 & 13 & 33 & 10 \\
\hline II & 40 & 19 & 17 & 39 & 14 \\
\hline III & 28 & 13 & 12 & 22 & 6 \\
\hline
\end{tabular}

CRT, chemoradiotherapy; RT, radiotherapy; cCRT, completed chemoradiotherapy; rCRT, reduced chemoradiotherapy; dCRT, discontinued chemoradiotherapy; cRT, completed radiotherapy; uRT, unfinished radiotherapy; TNM, tumor-node-metastasis.

Treatment progress. A total of 124 patients did not receive systemic chemotherapy and underwent RT alone due to comorbidities, personal choice or other reasons; 30 of these patients $(24.2 \%)$ did not complete the planned RT regimen [unfinished RT group (uRT)] and 94 patients $(75.8 \%$ ) completed the planned RT regimen (cRT group). A total of 188 patients received comprehensive treatment with CRT, of whom $42(22.3 \%)$ discontinued the treatment [discontinued CRT group (dCRT)], 47 (25\%) underwent a reduction in the dose of chemotherapy or the planned radiation dose by $10 \%$ after 1 cycle of CRT [CRT reduction group (rCRT) and $99(52.7 \%)$ completed the treatment [completed chemotherapy group (cCRT)]. There were no significant differences among the T, $\mathrm{N}$ and clinical staging data distribution in each group (Table II). With regard to the proportion of patients who completed CRT and RT, the difference was statistically significant $\left(\chi^{2}=16.967 ; \mathrm{P}=0.000\right)$.

Additionally, a statistical analysis was performed for age, PS and Charlson score of 42 patients from the dCRT group, 47 patients from the rCRT group and 30 patients from the uRT group (Table III). In the dCRT group, the differences between patients with a PS of $\geq 2$ and those with a PS of $<2$ were not statistically significant $\left(\chi^{2}=2.914 ; \mathrm{P}=0.088\right)$, whereas the differences between patients with a Charlson score of $\geq 2$ and those with a score of $<2$ were statistically significant $\left(\chi^{2}=33.599 ; \mathrm{P}=0.000\right)$. Patients aged $\geq 85$ years accounted for $90.9 \%$ of the dCRT group (20/22) and patients aged $<85$ years accounted for $13.3 \%$ (22/166) of the group; the difference between these two age groups was statistically significant $\left(\chi^{2}=67.521 ; \mathrm{P}=0.000\right)$. In the rCRT group, the difference between patients with a PS of $\geq 2$ and those with a PS of $<2$ was statistically significant $\left(\chi^{2}=13.061 ; \mathrm{P}=0.000\right)$. The difference between the patients with a Charlson score of $\geq 1$ and those with a score of 0 was statistically significant $\left(\chi^{2}=6.596 ; P=0.01\right)$. Patients ranging in age from 75 to
79 years accounted for $22.1 \%$ (25/113) of the rCRT group, whereas patients aged 80-84 years accounted for $37.7 \%$ (20/53) of the rCRT group, with a statistically significant difference between these two age groups $\left(\chi^{2}=4.450 ; \mathrm{P}=0.035\right)$.

Short- and long-term response evaluation. The CR rates in the chemotherapy, RT and reduced-dosage groups were 52.5 (52/99), 38.3 (36/94) and 46.8\% (22/47), respectively. The comparison of the CR rate of the CRT group to that of the RT group revealed a statistically significant difference $\left(\chi^{2}=3.935 ; \mathrm{P}=0.047\right)$. The comparison of the $\mathrm{CR}$ rate among the chemotherapy and dosage-reduction groups revealed no statistically significant difference $\left(\chi^{2}=0.417 ; \mathrm{P}=0.519\right)$.

A statistical analysis of overall survival (OS) was performed in the rCRT, cCRT and RT groups at 1, 3 and 5 years of follow-up, respectively. At 1, 3 and 5 years, the OS was $59.6(n=28), 17.0(n=8)$ and $10.6 \%(n=5)$, respectively, in the rCRT group; $60.6(n=60), 17.2(n=17)$ and $11.1 \%(n=11)$, respectively, in the cCRT group; and $58.5(n=55), 2.1 \quad(n=2)$ and $0 \%(0)$, respectively, in the RT group. Furthermore, the comparison of the OS rates between the rCRT and cCRT groups revealed no statistically significant difference $(\mathrm{P}=0.905,0.982$ and 0.932 , respectively). There were no significant differences in the OS rate between the RT and the CRT groups in the first year $(\mathrm{P}=0.767)$; however, the OS rates at 3 and 5 years were significantly lower compared to those in the other two groups $(\mathrm{P}=0.000$ and 0.001$)$.

Toxicity reactions. Adverse toxicity reactions $\geq$ grade 3 or reactions that were life-threatening were recorded. The frequency of toxicity reactions was $30.3,14.9,42.9,18.1$ and $23.3 \%$ in the cCRT (30/99), rCRT (7/47), dCRT (18/42), RT (17/94) and uRT (7/30) groups, respectively. Among these, the incidence of adverse toxicity reactions $\geq$ grade 3 in the dCRT group was significantly higher compared to that in the other groups; the 
Table III. Patient stratification by performance status, Charlson score and age.

\begin{tabular}{lrrr}
\hline Variables & dCRT, n/total $(\%)$ & rCRT, n/total $(\%)$ & uRT, $\mathrm{n} /$ total $(\%)$ \\
\hline Performance status & & & \\
0 & $10 / 51(19.6)$ & $8 / 51(15.6)$ & $2 / 9(22.2)$ \\
1 & $22 / 108(20.4)$ & $24 / 108(22.2)$ & $15 / 71(21.1)$ \\
$\geq 2$ & $10 / 29(34.5)$ & $15 / 29(51.7)$ & $13 / 44(29.5)$ \\
Charlson score & & & \\
0 & $4 / 36(11.1)$ & $3 / 36(8.3)$ & $0 / 6(0)$ \\
1 & $20 / 126(15.9)$ & $35 / 126(27.7)$ & $2 / 20(10.0)$ \\
$\geq 2$ & $17 / 26(65.4)$ & $9 / 26(34.6)$ & \\
Age (years) & & & $17 / 76(22.4)$ \\
$75-79$ & $14 / 113(12.4)$ & $25 / 113(22.1)$ & $8 / 35(22.9)$ \\
$80-84$ & $8 / 53(15.1)$ & $20 / 53(37.7)$ & $3 / 9(33.0)$ \\
$85-89$ & $17 / 19(89.5)$ & $2 / 19(10.5)$ & $2 / 4(50.0)$ \\
$\geq 90$ & $3 / 3(100.0)$ & - &
\end{tabular}

dCRT, discontinued chemoradiotherapy; rCRT, reduced chemoradiotherapy; uRT, unfinished radiotherapy.

Table IV. Causes of death in the CRT and RT treatment groups.

\begin{tabular}{|c|c|c|c|c|c|}
\hline \multirow[b]{2}{*}{ Causes of death } & \multicolumn{3}{|c|}{ CRT (n=172) } & \multicolumn{2}{|c|}{$\mathrm{RT}(\mathrm{n}=124)$} \\
\hline & cCRT, n (\%) & rCRT, n (\%) & dCRT, n (\%) & cRT, n (\%) & uRT, n (\%) \\
\hline \multicolumn{6}{|l|}{ Tumor } \\
\hline Recurrence & $15(17.0)$ & $8(19.0)$ & $28(66.7)$ & $16(17.0)$ & $22(73.3)$ \\
\hline Relapse & $30(34.1)$ & $14(33.3)$ & - & $30(31.9)$ & - \\
\hline Metastasis & $20(22.7)$ & $9(21.4)$ & - & $35(37.2)$ & - \\
\hline Relapse and metastasis & $5(5.7)$ & $3(7.2)$ & - & $4(4.3)$ & - \\
\hline Treatment-related & $2(2.2)$ & $1(2.4)$ & $8(19.1)$ & $3(3.2)$ & $1(3.3)$ \\
\hline Underlying disease & $13(14.8)$ & $3(7.2)$ & $5(11.9)$ & $4(4.3)$ & $6(20.0)$ \\
\hline Other & $3(3.4)$ & $4(9.5)$ & $1(2.3)$ & $2(2.1)$ & $1(3.3)$ \\
\hline
\end{tabular}

CRT, chemoradiotherapy; RT, radiotherapy; cCRT, completed chemoradiotherapy; rCRT, reduced chemoradiotherapy; dCRT, discontinued chemoradiotherapy; uRT, unfinished radiotherapy; cRT, completed radiotherapy.

difference was statistically significant between the cCRT and rCRT groups $\left(\chi^{2}=4.000 ; \mathrm{P}=0.046\right)$ and between the $\mathrm{RT}$ and CRT groups $\left(\chi^{2}=3.907 ; \mathrm{P}=0.048\right)$. Adverse toxicity reactions $\geq$ grade 3 did not differ significantly between the RT and the rCRT group $\left(\chi^{2}=0.226 ; \mathrm{P}=0.635\right)$.

Cause of death. During follow-up, a total of 296 deaths were recorded. The mortality rate due to tumor-related factors in the cCRT, rCRT, dCRT, RT and uRT groups was 79.5, 80.95, $66.7,90.4$ and $73.3 \%$, respectively (Table IV). The mortality rate due to underlying disease in the cCRT group was significantly higher compared with that in the other groups (14.8\%). Additionally, the mortality rate was significantly higher in the dCRT group compared with that in the other groups $(19.1 \%)$ and mortality due to distant metastasis in the RT alone group was also higher compared with that in the other groups $(37.2 \%)$.

\section{Discussion}

The improvements in general healthcare and increased life expectancy have resulted in an increased number of elderly patients with EC. It was previously reported that, at 75 years of age, life expectancy is $>10$ years (12) Therefore, provided there are no major comorbidities, elderly patients with EC may benefit from curative treatment. Our results indicated that CRT was an effective treatment for EC patients aged $\geq 75$ years. Our results also indicated that patient selection and the short- and long-term outcomes of CRT for elderly patients were considered acceptable, which is in accordance with the results reported in previous studies $(7,13)$. Our study mainly focused on those patients who required treatment discontinuation or a reduction in treatment dose.

Age and comorbidity were associated with significant difficulties during treatment, particularly in patients treated 
with CRT. Our results indicated that an age of 75 years did not affect the frequency of adverse events. It was previously reported that tolerance to 5-FU was the same in elderly and younger patients (14). Furthermore, a previous study on patients with advanced esophagogastric adenocarcinoma suggested that cisplatin-based chemotherapy toxicities did not increase with age (15). Those results indicated that age criteria alone may not suffice for the guidance of therapy and a better characterization of patients using the Charlson score is required. However, the number of studies on the safety and efficacy of selective CRT treatment in patients with EC aged $\geq 75$ years based on the Charlson score is limited.

In this study, we demonstrated that, based on the completion of treatment, the tolerance of patients who received RT alone was satisfactory and the completion rate was superior to that of patients who underwent CRT; our results were in accordance with the findings of previous studies $(1,2)$. In our study, the proportion of patients with a Charlson score of $\geq 2$ who discontinued their treatment was significantly higher in the dCRT group compared to that in the other groups, although there were no differences in the PS. Anderson et al (13) reported that there was a correlation between a Charlson score of $\geq 2$ and grade 3 or 4 toxicity. In the present study, the proportion of patients aged $\geq 85$ years in the dCRT group was significantly higher compared with that of patients aged $\leq 85$ years and the number of patients with $\geq 3$ grade toxicity in this group was also higher compared to that in the other groups. This suggested that chemotherapy in EC patients aged $\geq 85$ years should be administered with greater caution. No studies on the effects of CRT in EC patients aged $\geq 75$ years are currently available.

The comparison of patients with a Charlson score of 0 and those with a Charlson score of $\geq 1$ indicated that the proportion of patients who experienced rCRT was higher in the latter group. Additionally, in patients with a PS of $\geq 2$, the number who experienced rCRT was significantly higher compared to those with a PS of $\geq 1$. Patients with a Charlson score of $\geq 1$ experienced more adverse events of grade $\geq 2$ and a delay in undergoing chemotherapy (7). The comparison of patients aged $<80$ years with those aged $\geq 80$ years revealed that the proportion of patients experiencing a rCRT was higher among those aged $\geq 80$ years. Furthermore, the number of patients with toxicity reactions of grade $\geq 3$ was lower in the rCRT group compared to that in the cCRT group, but there were no significant differences with regards to $\mathrm{CR}$ and $\mathrm{OS}$. This regimen may be an appropriate adjustment for older EC patients who cannot tolerate standard therapy and exhibit a high comorbidity rate. Previous studies suggested that a PS of $<2$ was one of the factors affecting survival (7); according to the results of the present study, in the assessment of patient treatment, tolerance was not as sensitive as the Charlson score.

With regard to mortality, tumor-related factors were the primary cause; furthermore, the rate of distant metastasis in the RT alone group was higher compared with that in the CRT group. This was consistent with corresponding results from previous studies $(3,16)$. With the extension of the survival time in the cCRT group, the mortality rate due to underlying disease was higher compared to that in the other groups.

In conclusion, we demonstrated that CRT is an effective treatment for EC patients aged $\geq 75$ years; however, individualized treatment is recommended in accordance with further age regrouping and pretreatment in line with the Charlson score. For patients aged $\geq 80$ years, a lower-dose therapy was found to be more effective and for those aged $\geq 85$ years, CRT should be administered with greater caution.

\section{References}

1. Cooper JS, Guo MD, Herskovic A, et al: Chemoradiotherapy of locally advanced esophageal cancer: long-term follow-up of a prospective randomized trial (RTOG 85-01). Radiation Therapy Oncology Group. JAMA 281: 1623-1627, 1999.

2. Minsky BD, Pajak TF, Ginsberg RJ, et al: INT 0123 (Radiation Therapy Oncology Group 94-05) phase III trial of combined-modality therapy for esophageal cancer: high-dose versus standard-dose radiation therapy. J Clin Oncol 20: 1167-1174, 2002.

3. Steyerberg EW, Neville B, Weeks JC and Earle CC: Referral patterns, treatment choices, and outcomes in locoregional esophageal cancer: a population-based analysis of elderly patients. J Clin Oncol 25: 2389-2396, 2007.

4. Ruol A, Portale G, Zaninotto G, et al: Results of esophagectomy for esophageal cancer in elderly patients: age has little influence on outcome and survival. J Thorac Cardiovasc Surg 133: 1186-1192, 2007.

5. Kleinberg L and Forastiere AA: Chemoradiation in the management of esophageal cancer. J Clin Oncol 25: 4110-4117, 2007.

6. Kosugi S, Sasamoto R, Kanda T, Matsuki A and Hatakeyama K: Retrospective review of surgery and definitive chemoradiotherapy in patients with squamous cell carcinoma of the thoracic esophagus aged 75 years or older. Jpn J Clin Oncol 39: 360-366, 2009.

7. Tougeron D, Di Fiore F, Thureau S, et al: Safety and outcome of definitive chemoradiotherapy in elderly patients with oesophageal cancer. Br J Cancer 99: 1586-1592, 2008.

8. Klabunde CN, Potosky AL, Legler JM and Warren JL: Development of a comorbidity index using physician claims data. J Clin Epidemiol 53: 1258-1267, 2000.

9. Therase P, Arbuck SG, Eisenhauer EA, et al: New guidelines to evaluate the response to treatment in solid tumors (RECIST Guidelines). J Natl Cancer Inst 92: 205-216, 2000.

10. Cox JD, Stetz J and Pajak TF: Toxicity criteria of the Radiation Therapy Oncology Group (RTOG) and the European Organization for Research and Treatment of Cancer (EORTC). Int J Radiat Oncol Biol Phys.31:1341-1346, 1995.

11. Trotti A, Byhardt R,Stetz J, et al: Common toxicity criteria: version 2.0. an improved reference for grading the acute effects of cancer treatment: impact on radiotherapy. Int J Radiat Oncol Biol Phys 47: 13-47, 2000.

12. Arias E: United States life tables, 2006. Natl Vital Stat Rep 58: 1-40, 2010.

13. Anderson SE, Minsky BD, Bains M, Hummer A, Kelsen D and Ilson DH: Combined modality chemoradiation in elderly oesophageal cancer patients. Br J Cancer 96: 1823-1827, 2007.

14. Popescu RA, Norman A, Ross PJ, Parikh B and Cunningham D: Adjuvant or palliative chemotherapy for colorectal cancer in patients 70 years or older. J Clin Oncol 17: 2412-2418, 1999.

15. Trumper M, Ross PJ, Cunningham D, et al: Efficacy and tolerability of chemotherapy in elderly patients with advanced oesophago-gastric cancer: a pooled analysis of three clinical trials. Eur J Cancer 42: 827-834, 2006.

16. Tougeron D, Hamidou J, Scotté M, Di Fiore F, Antonietti M, Paillot B and Michel P: Esophageal cancer in the elderly: an analysis of the factors associated with treatment decisions and outcomes. BMC Cancer 10: 510, 2010. 